\title{
Determining polar ionospheric electrojet currents from Swarm satellite constellation magnetic data
}

\author{
Cecilie Drost Aakjær ${ }^{*} \mathbb{D}$, Nils Olsen and Christopher C. Finlay
}

\begin{abstract}
We determine the strength and location of the ionospheric currents responsible for the polar electrojets from magnetic data collected by the Swarm satellite constellation on an orbit-by-orbit basis. The ionospheric currents are modelled using a simple, yet robust, method by a series of line currents at $110 \mathrm{~km}$ altitude (corresponding to the ionospheric E-layer) perpendicular to the satellite orbit, separated by $1^{\circ}$ (about $113 \mathrm{~km}$ ). We assess the reliability of our method, with the aim of a possible near-real-time application. A study of the effect of different regularization methods is therefore carried out. An $L_{1}$ model regularization of the second-order spatial differences, and robust treatment of the data (to account for non-Gaussian error distributions), yields the most encouraging results. We apply our approach to two three-weekly data periods in March 2014 (geomagnetic quiet conditions) and March 2015 (more disturbed conditions), respectively. Our orbit-by-orbit approach also allows the temporal evolution of the polar electrojets to be investigated. We find remarkable agreement of the ionospheric activity in Northern and Southern polar regions, with correlation exceeding 0.9 for periods longer than two days. Reliability of the approach is shown by three key results: (1) a common regularization parameter for all orbits with enough data coverage, (2) 0.95 squared coherence with the Auroral Electrojet index, and (3) 0.97 squared coherence is found between the side-by-side flying satellites, Alpha and Charlie, indicating a method invariant to small changes in data input. All these results indicate a possible automated near-real-time application.
\end{abstract}

Keywords: Geomagnetism, Field modelling, lonosphere, Swarm

\section{Introduction}

Geomagnetic reference models provide a good description of the main parts of Earth's magnetic field, including contributions from the core and crust, as well as the large-scale magnetospheric (e.g. Finlay et al. 2016; Lesur et al. 2010; Olsen et al. 2014). Non-ionospheric field (e.g. Sabaka et al. 2004, 2015) contributions are also well described by modern models. The situation is, however, different in the polar regions, where the large temporal and spatial variability of electric currents in the polar ionosphere makes their description difficult, and their prediction almost impossible.

*Correspondence: cda@space.dtu.dk

Division of Geomagnetism, DTU Space, Technical University of Denmark, Diplomvej 371, Kongens Lyngby, Denmark
These ionospheric currents give rise to a variety of important space weather effects, influencing the performance and reliability of spaceborn and ground-based technological systems. Problems in ground-based systems occur for instance due to the secondary, Earthinduced, electric fields and corresponding currents. For directional drilling in polar regions disturbances in the magnetic field caused by strong ionospheric currents may hamper accurate well positioning (Poedjono et al. 2013). Ionospheric currents may also lead to increased drag on low-altitude spacecraft (Liu and Lühr 2005; Pirjola et al. 2005). Better understanding of the time-space structure of polar ionospheric currents and in particular their improved modelling are therefore of great importance, not only for advances in fundamental space research but also regarding practical applications. 
There are two major constituents to magnetic disturbance fields at polar latitudes: (1) electrical currents in the ionospheric E-layer (at an altitude of about $110 \mathrm{~km}$ ) form the polar electrojets (PEJs) and (2) currents flowing along field lines of the ambient magnetic field feeding the PEJs by connecting the ionosphere and the magnetosphere. These so-called field-aligned currents (FACs) result in large magnetic field disturbances at satellite altitude, in particular in the east-west magnetic field component.

The PEJs have been successfully estimated from ground magnetic data (e.g. Amm 1997; Friis-Christensen et al. 1985; Kamide et al. 1981; Richmond et al. 1998). Indices monitoring the electrojet activity, such as the Auroral Electrojet (AE) index (Sugiura and Davis 1966) have been developed. These indices provide important information on the state of the polar ionosphere, but are restricted by the positions of the magnetometer stations. Since primarily stations from the Northern Hemisphere are used, the resulting indices mainly reflect PEJ activity in that hemisphere.

From magnetic ground observations it is only possible to estimate the equivalent currents, e.g. (hypothetic) horizontal ionospheric currents that would cause the same magnetic field disturbance as the observed one. Magnetic observations taken at satellite height in addition allow the estimation of the FACs, which is a clear advantage compared to ground observations. Furthermore, satellites allow access to the entire, mainly north-south-directed, current density profile, in contrast to what is possible from ground observations, and thereby provide a better description of the currents position and magnitude as a function of latitude. The global coverage of satellite data also enables interhemispheric comparisons. With these advantages in mind, several studies of ionospheric currents have been conducted using satellite measurements from, e.g., the Magsat, Ørsted, and CHAMP satellites (Olsen 1996; Ritter et al. 2003).

The magnetic field produced by an electric current is, at least in the vicinity of the current, always perpendicular to the current direction. This means that the magnetic field caused by FACs is perpendicular to the field line and hence not observable in the magnetic component parallel to the field line. As a consequence, the magnetic field intensity, $F=|\mathbf{B}|$, which by definition is the field component parallel to the magnetic field, is only marginally affected by FACs. Field intensity, $F$, is, however, sensitive to contributions from the horizontal currents in the ionospheric E-layer that form the PEJs. Observations of $F$ collected by satellites can therefore be used to determine these currents.

A model study of the PEj's using Magsat scalar magnetic satellite data and a current model consisting of a series of line currents perpendicular to the satellite track was presented by Olsen (1996) and later applied to CHAMP satellite data by Ritter et al. (2003) and Ritter et al. (2004). The use of scalar magnetic satellite data is a simplification compared to using the full vector data, resulting in similar results to a ground magnetic chain. The line current method has been applied to multi-satellite data by Olsen et al. (2002) and Moretto et al. (2002).

Juusola et al. (2006) proposed an alternative approach for monitoring the auroral activity from magnetic satellite data. They worked with a $1 \mathrm{D}$ version of the $2 \mathrm{D}$ Spherical Elementary Current System (SECS) method developed by Amm (1997) for application to CHAMP satellite vector data. By using vector data they were able to estimate not only the horizontal currents but also the FACs. The use of vector data in the 1D SECS method may, however, introduce both a complication in computation and an error source due to stronger contamination by unwanted sources such as the FAC in the radial magnetic vector component compared to only using measurements of the magnetic field intensity.

A simple approach to determine the location and strength of the PEJs from magnetic satellite data was presented by Vennerstrom and Moretto (2013). Their method corresponds roughly to finding the position and amplitude of the PEJs in the current profiles determined using the line current model of Olsen (1996).

With the aim of a possible near-real-time monitoring of the time-space structure of polar electrojet activity, we aim at developing a reliable and simple approach, while still estimating entire current profile along the satellite tracks. We therefore applied the line current model of Olsen (1996) to Swarm satellite magnetic data and investigated how different regularization methods affect the model results. By applying the method to Swarm magnetic data, we do not only gain new insights concerning the current system in the ionosphere, but also have the opportunity to explore previously unavailable results regarding longitudinal variations of the PEJs thanks to the unique constellation of the Swarm satellites.

The trio of Swarm satellites (e.g. Friis-Christensen et al. 2008) has been in orbit since 22 November 2013. Two of the satellites, called Alpha and Charlie, fly side by side at an altitude of about $450 \mathrm{~km}$ (as of January 2016) in a near-polar orbit of inclination $87.4^{\circ}$ with an east-west separation of $1.4^{\circ}$ in longitude (corresponding to about $160 \mathrm{~km}$ at the equator). The third satellite, Bravo, has an orbital inclination of $88^{\circ}$ and is flying at an altitude of approximately $520 \mathrm{~km}$. The different orbital inclination of the satellites results in different drift rates in local time (LT). The Bravo satellite in November 2015 (i.e. two years after launch) measured the magnetic field at a local time $2.6 \mathrm{~h}$ ahead of the lower satellite pair Alpha and Charlie. 
Each of the three satellites carry, amongst other instruments, an absolute scalar magnetometer (ASM) for measuring the magnetic field intensity $F$, a vector fluxgate magnetometer (VFM) measuring the three vector components of the magnetic field, and a triple-head star imager to determine orientation.

The first part of the paper presents the chosen model parametrization along with a description of the model estimation scheme and an exploration of various regularization methods. Next we present results from a single orbit crossing and then move on to consider time dependence in three weeks of data for both the Northern and Southern Hemisphere. We also compare our results with the Auroral Electrojet index, a ground-based measure of PEJ activity. Finally, results obtained with data from all three Swarm satellites are compared, and the effects of secondary, Earth-induced, currents are discussed.

\section{Model parameterization}

We describe the polar ionospheric currents by a series of line currents placed at an altitude of $110 \mathrm{~km}$ in the ionospheric E-layer, perpendicular to the satellite track and separated in horizontal (along-track) direction by $\Delta=113 \mathrm{~km}$ corresponding to $1^{\circ}$. The geometry of the model is shown in Fig. 1. The magnetic field due to ionospheric E-layer currents is a Laplacian potential field at satellite altitude, and thus the strength of the magnetic field, and the spatial structure of the ionospheric currents, depends on the distance to the measurements. A typical rule of thumb in this case is that one cannot resolve structure of scale smaller than half the distance to the measurements. Since the Swarm satellites measure at a height of minimum $340 \mathrm{~km}$ above the ionosphere, it is hardly possible to distinguish between a continuous current distribution and a series of discrete line currents separated by $1^{\circ}$ (corresponding to about $113 \mathrm{~km}$ ). We therefore use a series of discrete line current separated by $1^{\circ}$.

As mentioned previously, electric currents at satellite altitudes are predominantly confined to flow parallel to the field lines of the ambient magnetic field $\mathbf{B}_{0}$ due to the nearly vanishing transverse electrical conductivity in this region. These FACs do, at least to first order, not contribute to the magnetic field component $F=B_{\|}$parallel to the ambient field $\mathbf{B}_{0}$. In the ionospheric E-layer, however, the transverse conductivity is comparably large, resulting in horizontal currents causing magnetic signatures that do not contribute to $F=B_{\|}$. The magnetic field intensity $F$ can therefore be used to investigate the horizontal currents flowing in the ionospheric E-layer. Although this is strictly true only for a uniform ambient field $\mathbf{B}_{0}$, FACs give, even for a real distribution of currents, only a very small contribution to $F$.

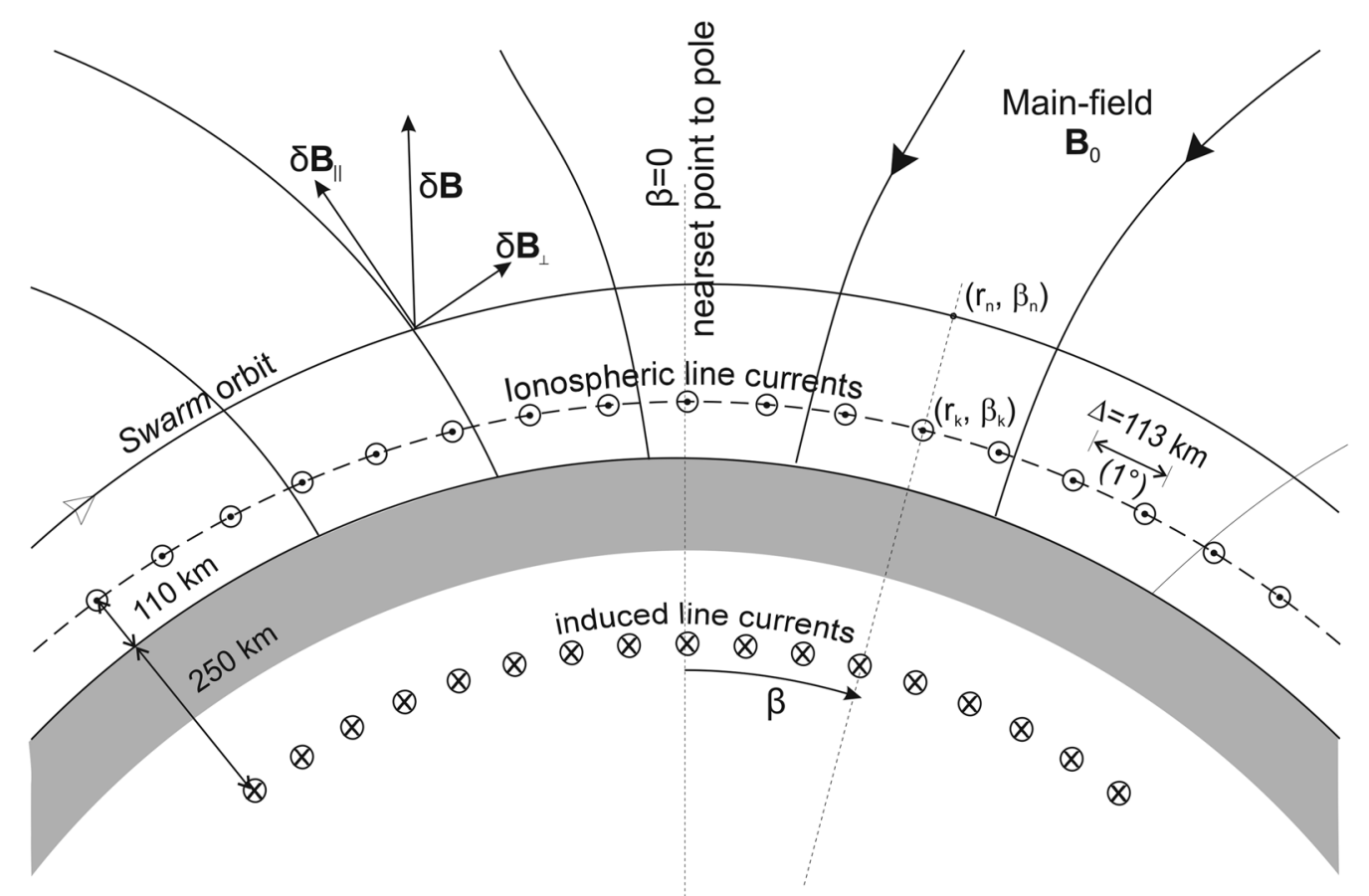

Fig. 1 Geometry of the model 
As input data we use field intensity anomaly values $\delta F=F^{\text {obs }}-F^{\text {mod }}$ obtained from satellite observations of the Mag LR $1 \mathrm{~Hz}$ L1b magnetic field intensity, $F^{\text {obs }}$ downsampled to $10 \mathrm{~s}$, after removal of model values, $F^{\text {mod }}$, given by the CHAOS-5 field model (Finlay et al. 2015). This model provides estimates for the core and crustal field contributions as well as the contributions from the large-scale magnetospheric field, the time dependence of which is given by the $R C$ index, which describes the strength of the magnetospheric ring current (Olsen et al. 2014).

The contribution from one single line current to the magnetic field intensity $\delta F$ is given, following Olsen (1996), by

$$
\begin{aligned}
\delta F_{n} & =\frac{\mu_{0}}{2 \pi} \frac{\xi_{n, k} \cos I_{n}+\eta_{n, k} \sin I_{n}}{\xi_{n, k}^{2}+\eta_{n, k}^{2}} \cdot j_{k} \\
\eta_{n, k} & =r_{k} \sin \left(\beta_{n}-\beta_{k}\right) \\
\xi_{n, k} & =r_{n}-r_{k} \cos \left(\beta_{n}-\beta_{k}\right)
\end{aligned}
$$

where $\mu_{0}=4 \pi \cdot 10^{-7} \mathrm{Vs} / \mathrm{Am}$ is vacuum magnetic permeability, $I_{n}$ is the magnetic inclination of the main field model, $\mathbf{B}_{0}$, at the satellite location $\left(r_{n}, \beta_{n}\right)$, and $j_{k}$ is the amplitude of the $k$ th line current at location $\left(r_{k}, \beta_{k}\right)$. $\eta_{n}$ and $\xi_{n}$ measure the radial and horizontal distance, respectively. $\beta_{n}$ and $\beta_{k}$ are along-track arc parameters describing the distance from the closest approach to the magnetic North geomagnetic pole (or South geomagnetic pole) for the observation locations and line current locations, respectively, as shown in Fig. 1.

The magnetic field disturbance caused by a superposition of the contributions from single line currents described by Eq. (1) results in a model of the form

$$
\boldsymbol{d}=\boldsymbol{G m}
$$

where $\boldsymbol{d}$ is the data vector consisting of the $N$ observations, $\delta F_{n}$, with $n=1, \ldots, N, \boldsymbol{m}$ is the vector of the $M$ model parameters (the line current amplitudes), $j_{k}$, with $k=1, \ldots, M$, and $\boldsymbol{G}$ is the design matrix of size $N \times M$ with elements

$$
g_{n, k}=\frac{\mu_{0}}{2 \pi} \frac{\xi_{n, k} \cos I_{n}+\eta_{n, k} \sin I_{n}}{\xi_{n, k}^{2}+\eta_{n, k}^{2}} .
$$

The sheet current density $J\left(\beta_{k}\right)$ is estimated from the model parameters by dividing the line current amplitudes $j_{k}$ (the model parameters) by the distance, $\Delta \beta=113 \mathrm{~km}$, between the line currents that corresponds to $1^{\circ}$ at $110 \mathrm{~km}$ altitude. Since the spacing is equidistant in $\beta$, the sheet current density is simply found as

$$
J\left(\beta_{k}\right)=\frac{j_{k}}{\Delta \beta}=\frac{j_{k}}{113 \mathrm{~km}}
$$

\section{Model estimation}

The $M$ model parameters $j_{k}$ are estimated by solving the linear inverse problem described in Eq. (2). An iteratively reweighted least squares (IRLS) approach with Huber weights (Constable 1988; Huber 1964) is used in order to handle a possibly non-Gaussian data error distribution. Although formally overdetermined (since $N>M$ ), the problem is ill-conditioned, and thus, the use of unconstrained least squares results in huge variations of the amplitudes of neighbouring line currents. In order to avoid this instability, we adopt a regularization approach (Aster et al. 2005; Menke 2012).

We carried out tests on a range of different regularization norms including: zeroth-order Tikhonov regularization (Tikhonov 1963), i.e. minimizing the sum of the squares of the line current strengths; higher-order Tikhonov regularization (Aster et al. 2005) considering finite differences of the line current strengths in the along-track direction; maximum entropy regularization (Jackson et al. 2007) as well as the use of L1 rather than L2 norms (Farquharson and Oldenburg 1998). Below we focus on the two following approaches that were found to perform well:

1 Minimization of an $L_{2}$ norm of the model parameters [i.e. zeroth-order Tikhonov regularization, see Aster et al. (2005)] and a Huber-weighted misfit measure. This is implemented through an IRLS technique, where the model at the $(i+1)$ th iteration is determined by

$$
\boldsymbol{m}_{i+1}^{\boldsymbol{L}_{2}}=\left(\boldsymbol{G}^{T} \boldsymbol{W}_{i}^{d} \boldsymbol{G}+\alpha^{2} \boldsymbol{I}\right)^{-1} \boldsymbol{G}^{T} \boldsymbol{W}_{i}^{d} \boldsymbol{d} .
$$

Here $\boldsymbol{I}$ is the identity matrix of size $M \times M, \alpha^{2}$ is a parameter controlling the strength of the regularization, and $\boldsymbol{W}_{i}^{d}$ is the data weight matrix. $\alpha^{2}$ is adjusted to achieve the desired trade-off between data misfit and model complexity. Its value and choice thereof is discussed further in the section "Choice of regularization parameter, $\alpha^{2}$ ". The Huber-weighted misfit approach allows non-Gaussian errors to be handled by applying small weights to outliers through a series of iterations. $W_{i}^{d}$ is the Huber weight matrix for the $i$ th iteration with diagonal elements $w_{i, n}=\min \left(\frac{c \sigma_{i}}{|\Delta \delta F|}\right) \cdot c$

is here a constant typically $1.5, \sigma$ is the standard deviation of the model residuals $\Delta \delta F=\delta F^{o b s}-\delta F^{\mathrm{mod}}$, where $\delta F^{\mathrm{mod}}$ are the model predictions of the observations. This solution will be referred to below as the $L_{2}$ norm solution.

2 Minimization of an $L_{1}$ norm of the second-order differences of model parameters and a Huber-weighted 
misfit measure. This is also implemented via IRLS via the scheme

$$
\boldsymbol{m}_{i+1}^{\boldsymbol{L}_{\mathbf{1}}}=\left(\boldsymbol{G}^{T} \boldsymbol{W}_{i}^{d} \boldsymbol{G}+\alpha^{2} \boldsymbol{D}^{T} \boldsymbol{W}_{i}^{m} \boldsymbol{D}\right)^{-1} \boldsymbol{G}^{T} \boldsymbol{W}_{i}^{d} \boldsymbol{d} .
$$

$\boldsymbol{D}$ is the second-order finite-difference operator, controlling the second-order difference minimization of the model parameters (Aster et al. 2005), and $W_{i}^{m}$ is a reweighting matrix implementing the $L_{1}$ minimization of the model parameters (Farquharson and Oldenburg 1998). The elements of the diagonal matrix $\boldsymbol{W}_{i}^{m}$ are found using Ekbloms measure, where $\left(W_{k k}^{m}=\left(j_{k}^{2}+\epsilon^{2}\right)^{-1 / 2}\right.$ Ekblom (1987). $\epsilon$ ensures a nonsingular solution at $j_{k}=0$. A sufficiently small value of $\epsilon$ compared to the magnitude of the model parameters was chosen. Note that $\boldsymbol{D}^{T} \boldsymbol{W}_{i}^{m} \boldsymbol{D}$ is non-dimensionless and $\alpha^{2}$ in Eq. 6 will therefore have a different unit than in Eq. 5. This solution will be referred to below as the $L_{1}$ norm solution.

\section{Results and discussion}

\section{Examples of geomagnetically quiet and disturbed periods}

We first illustrate our approach with a few examples, representing geomagnetic quiet and disturbed conditions, respectively. We have chosen Swarm Alpha orbit no. 6248 on 4 January 2015 13:36 to 14:01 UT, corresponding to a magnetic local time (MLT) of midnight around $60^{\circ}$ Quasi-Dipole (QD) latitude. Since ionospheric conductivity and currents are guided by the geometry of Earth's main field, it is advantageous to describe the currents using magnetic coordinates like the QD latitude (Richmond 1995). This was a moderately disturbed period, with index of global geomagnetic activity $\mathrm{Kp}=4^{+}$. As an example of quiet-time conditions, we have chosen orbit no. 6493 from 20 January 12:37 to 13:02 UT, at a similar MLT but with a Kp index of $0^{+}$.

The top row of Fig. 2 shows the observations of magnetic field intensity $F$, and the obtained data fit, the middle row shows the corresponding model residuals, and the bottom row presents the estimated sheet current density, $J$, for the disturbed day (left), and the quiet day (right).

Shown in green is the observed magnetic field signature $\delta F$ (after removal of core, crust, and magnetospheric contributions), along with model predictions obtained using $L_{1}$ (black) and $L_{2}$ (red) norm regularization. A simple least squares solution with zeroth-order Tikhonov regularization is given in blue for comparison. Both regularization schemes, with $\alpha^{2}=6.4 \cdot 10^{-15} \mathrm{nT} \cdot \mathrm{A}^{-2}\left(L_{1}\right)$ and $\alpha^{2}=3.6 \cdot 10^{-27} \mathrm{nT} \cdot \mathrm{A}^{-2}\left(L_{2}\right)$, are able to produce an extremely good fit to the observed field intensity. The chosen values of $\alpha^{2}$ are discussed in the section "Choice of regularization parameter, $\alpha^{2}$.

The variance ratios,

$$
\frac{\sigma_{\Delta \delta F}^{2}}{\sigma_{\delta F}^{2}}
$$

where $\Delta \delta F$ are the residuals between observations and the model predictions for the $L_{1}$ or for the $L_{2}$ solution are found to: $4.1 \cdot 10^{-6}\left(L_{1}\right)$ and $9.2 \cdot 10^{-6}\left(L_{2}\right)$ for the disturbed day (orbit 6248), and $39 \cdot 10^{-6}\left(L_{1}\right)$ and $67 \cdot 10^{-6}$ $\left(L_{2}\right)$ during quiet conditions (orbit 6493). This reveals that $L_{1}$ regularization results in slightly lower misfit than $L_{2}$ regularization, although both approaches describe almost all of the variance in the measurements. The small model residuals, $\Delta \delta F$, given in the middle row of Fig. 2 support the very low variance ratios with residuals lower than $1 \mathrm{nT}(\approx 0.5 \%$ of the signal strength) for the disturbed day.

Considering the mean variance ratios for 1000 orbits between 28 December 2014 to 3 March 2015 (orbit nos. 6142 to 7142 ) for satellite Swarm Alpha, we find that the $L_{1}$ solution in general gives a better description (mean variance ratio of $120 \cdot 10^{-6}$ ) compared to the $L_{2}$ solution $\left(400 \cdot 10^{-6}\right)$. This tells us that the model gives a very good data fit not only for the single orbits presented above, but for all tested orbits at least on average. The largest values of the variance ratio of the 1000 tested orbits was $820 \cdot 10^{-6}$ for the $L_{1}$ solution and $2000 \cdot 10^{-6}$ for the $L_{2}$ solution. Thus even for the orbits with the worst data fits (highest variance ratios), the model still performs very well. The orbits with highest variances are mainly from quiet days, since on disturbed days the signal amplitude is larger compared to the noise level.

The sheet current densities, $J$, are presented in the bottom row of Fig. 2 for both the $L_{1}$ (black) and $L_{2}$ (red) norm inversions as a function of QD latitude. A positive current is defined as a current flowing from midnight to noon (sunward). Compared to the reference least squares zeroth-order Tikhonov solution (blue curve), there are no major differences between the sheet current densities from $L_{1}$ (black curve) and $L_{2}$ (red curve) norm regularization. We expect that the sheet current densities are weak or absent where there are no currents (e.g. in non-polar regions) and not wildly fluctuating. The $L_{1}$ solution correctly estimates the currents outside the polar region (auroral oval) to be zero, or very close to zero, while the $L_{2}$ solution displays non-physical small-scale oscillations around zero. These cannot be damped sufficiently by increasing $\alpha^{2}$ without also reducing the amplitude of the peak of the sheet current density. The results obtained 

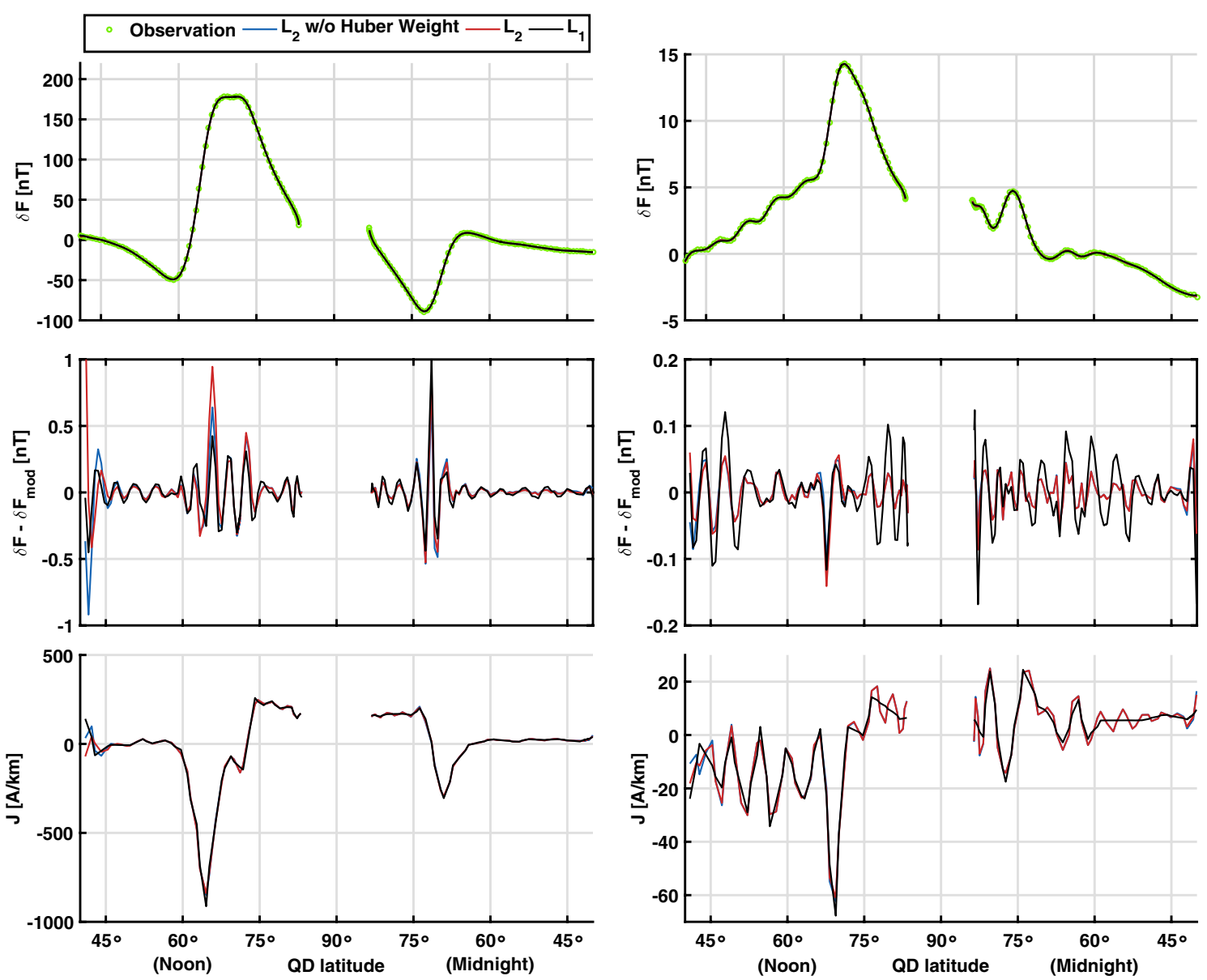

Fig. 2 Top row shows observations, $\delta F$, in green along with the model predictions, $\delta F^{\text {mod }}$, for three solution methods, $L_{1}$ norm (black), $L_{2}$ norm (red), and $L_{2}$ norm without Huber-weighted misfit (blue). The middle row shows the model residuals, $\Delta \delta F$, for the same inversion methods, and the bottom row shows the corresponding sheet current densities, J, again for all three methods. Data are from satellite Alpha for 2 days: (left column) orbit 6248 on 04.01.2015 13:36 to 14:01 UT, corresponding to a magnetic local time (MLT) of midnight around $60^{\circ}$ magnetic latitude (disturbed, $\mathrm{Kp}=4^{-}$) and (right column) orbit 6493 on 20.01.2015 12:37 to 13:02 UT, a similar MLT (quiet, $\mathrm{Kp}=0^{+}$). The sheet current densities are found using $\alpha^{2}=6.4 \cdot 10^{-15} \frac{\mathrm{nT}^{2}}{\mathrm{~A}}$ for the $L_{1}$ norm solution and $\alpha^{2}=3.6 \cdot 10^{-27} \frac{\mathrm{nT} \mathrm{T}^{2}}{\mathrm{~A}^{2}}$ for the $L_{2}$ norm solutions. A positive current flows from midnight to noon (sunward)

with the $L_{1}$ norm, therefore, give a representation of the model parameters slightly closer to what we expect on physical grounds, compared to the results found in the $L_{2}$ norm solution.

The simple least square zeroth-order Tikhonov regularization solution (blue curve in Fig. 2) gives a rather similar solution as the $L_{2}$ norm solution for these orbits, which also involves a Huber-weighted misfit measure. We prefer, however, to use the latter since the distribution of residuals (data minus model predictions) is non-Gaussian.

A similar argument also applies on going from $L_{2}$ to $L_{1}$ norm regularization. When solving with an $L_{2}$ regularization norm, we assume that the model parameters are Gaussian distributed. The distribution of the obtained model parameters is highly non-Gaussian, in particular shown in long tails; in this case it is more consistent to use an $L_{1}$ norm regularization scheme.

\section{Choice of regularization parameter, $\alpha^{2}$}

Both the $L_{2}$ and $L_{1}$ norm regularization methods require specification of a regularization parameter $\alpha^{2}$. The choice is a trade-off between goodness of data fit and model complexity. Too small values of $\alpha^{2}$ will result in interpretation of non-physical noise, while a value too large leads to a model for which the desired signal is suppressed, resulting in a decreased data fit. So the question is: when does damping regularization of a non-physical noise become damping of a physical signal?. One way to objectively choose $\alpha^{2}$ is by plotting the norm of the 
model vector, $\|\boldsymbol{m}\| / \max (\|\boldsymbol{m}\|)$ (individually normalized to the range between 0 and 1 for better comparison between different orbits) versus the norm of data misfit, $\| \mathbf{G} \boldsymbol{m}-\boldsymbol{d}^{\mathbf{o b s}}||$ for various values of the regularization parameter $\alpha^{2}$.

The individually normalized norm of the model vector as a function of $\alpha^{2}$ and data misfit is shown in a socalled L-curve (Hansen 1992) in Fig. 3 for the six example orbits. The L-curves are based on the model parameters obtained using the $L_{1}$ norm solution. The chosen orbits (nos. 200 (orange), 2000 (yellow), 3334 (dark blue), 6248 (light green), 6493 (light blue), and 8000 (purple)) represent orbit crossings for both disturbed and quiet times.

The range of $\alpha^{2}$ was chosen between $10^{-16} \mathrm{nT} \cdot \mathrm{A}^{-2}$ and $10^{-12} \mathrm{nT} \cdot \mathrm{A}^{-2}$. According to Hansen (1992), the optimal choice of $\alpha^{2}$ is in, or near, the corner of the L-curve. The corner value of $\alpha^{2}=1.2 \cdot 10^{-15} \mathrm{nT} \cdot \mathrm{A}^{-2}$ (red) seems to provide a good balance between the model misfit and complexity, but a visual inspection of the data fit and current profile revealed unwanted small-scale oscillations. This value was therefore increased slightly to obtain the final choice of $\alpha^{2}=6.4 \cdot 10^{-15} \mathrm{nT} \cdot \mathrm{A}^{-2}$ (approximately the purple marked value, $6.3 \cdot 10^{-15} \mathrm{nT} \cdot \mathrm{A}^{-2}$ ). The fact that the chosen $\alpha^{2}$ stays in almost the same position of the L-curve for different classes of $\mathrm{Kp}$ indicates that a common choice in $\alpha^{2}$ is possible for different geomagnetic activity conditions.

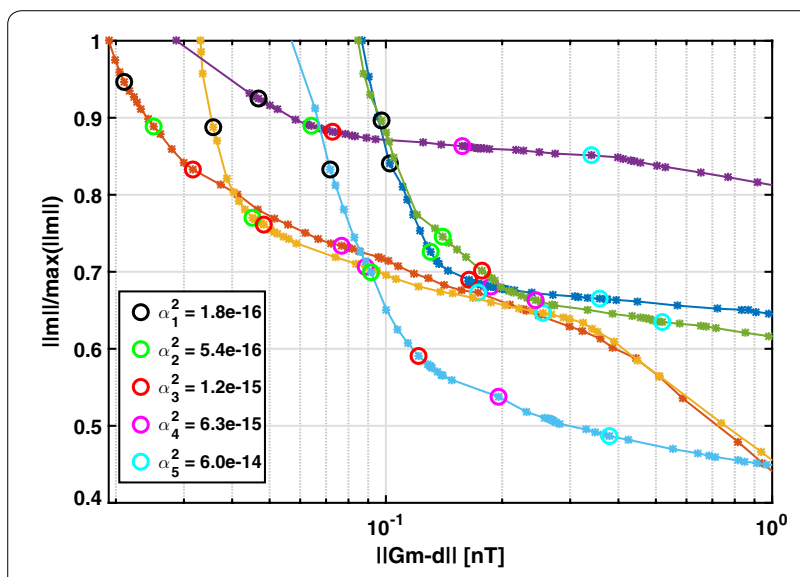

Fig. 3 Normalized L-curves for 6 individual orbits: orbit no. 200 with Kp index of $1^{-}$(orange), orbit no. 2000 with Kp index of $1^{+}$(yellow), orbit no. 3334 with Kp index of $3^{-}$(dark blue), orbit no. 6248 with Kp index of $4^{-}$(light green), orbit no. 6493 with Kp index of $0^{+}$(light blue), and orbit no. 8000 with Kp index of $1^{+}$(purple). Five

different $\alpha^{2}$ s are highlighted to show how changes in complexity and model fit change with changing values of $\alpha^{2}, \alpha_{1}^{2}=1.8 \cdot 10^{-16} \frac{\mathrm{nT}^{2}}{\mathrm{~A}}$,

$\alpha_{2}^{2}=5.4 \cdot 10^{-16} \frac{n T^{2}}{\mathrm{~A}}, \alpha_{3}^{2}=1.2 \cdot 10^{-15} \frac{\mathrm{nT}^{2}}{\mathrm{~A}}, \alpha_{4}^{2}=6.3 \cdot 10^{-15} \frac{\mathrm{nT}}{\mathrm{A}}$, and

$\alpha_{5}^{2}=6.0 \cdot 10^{-14} \frac{n T^{2}}{\mathrm{~A}}$
The preferred $\alpha^{2}$ will not only depend on the choice of regularization norm, but also on the number of observations, since a change in the size of $\boldsymbol{G}$ will result in a change in the weight between the two terms in the parenthesis of Eq. 5, and hence affect the choice in $\alpha^{2}$. Therefore, we need to be aware that orbits with large data gaps might need a different choice in $\alpha^{2}$.

\section{Temporal variations}

By looking at periods of several weeks, one can investigate the space-time evolution of ionospheric currents during both disturbed and quiet periods. Studying variations as a function of QD latitude, we can estimate where the currents are located, and whether the location changes with time and/or disturbance level. Figure 4 shows the sheet current densities as a function of time and QD latitude for a period of three weeks around spring equinox 2015 (10 to 31 March 2015). The top plot shows the Kp and AE indices; the Northern (middle panel), respectively, Southern (bottom panel) Hemisphere. Each plot is divided into a morning and an evening side. The sign of the current is defined as positive if directed from midnight to noon (sunward).

The oscillating gap around QD latitude 90 is not zero current, but comes from the satellite orbit inclination compared to the geographical pole and not the magnetic pole. The satellite will therefore only sometimes cross the magnetic pole. We find that the ionospheric electrojet current system is confined to the auroral oval $\left(\approx 70^{\circ}\right.$ to $80^{\circ} \mathrm{QD}$ latitude), and the strengths of the electrojets may therefore be estimated from the sheet current densities in this region. Densities of approximately $1 \mathrm{kA} / \mathrm{km}$ near the pole reveal polar cap currents, while the sheet current densities equatorward of the auroral oval are expected to be small. From the temporal development, we can see that deviations from zero at lower latitudes are largest during disturbed periods.

MLT changes by about $1.5 \mathrm{~h}$ at non-polar latitudes during the three weeks under consideration; at QD latitude $60^{\circ}$ it drifts on the evening side from approximately 20:30 to 19:00 for the example shown in Fig. 4. The period in question is mainly quiet, with low $\mathrm{Kp}$ and $\mathrm{AE}$ index values (see top plot), but indicates three more active intervals, around 13, 21, and 25 to 28 March 2015. Corresponding higher values of $\mathrm{Kp}$ are found for these periods.

Figure 4 reveals a large degree of consistency between the two hemispheres-strong sheet current densities are found in the Northern Hemisphere at the same times as in the Southern Hemisphere, as expected. The small interhemispheric differences may be due to the time difference of $45 \mathrm{~min}$ between the pole crossings. This will mainly be an issue during substorms, where changes in the current system can happen very rapidly. 

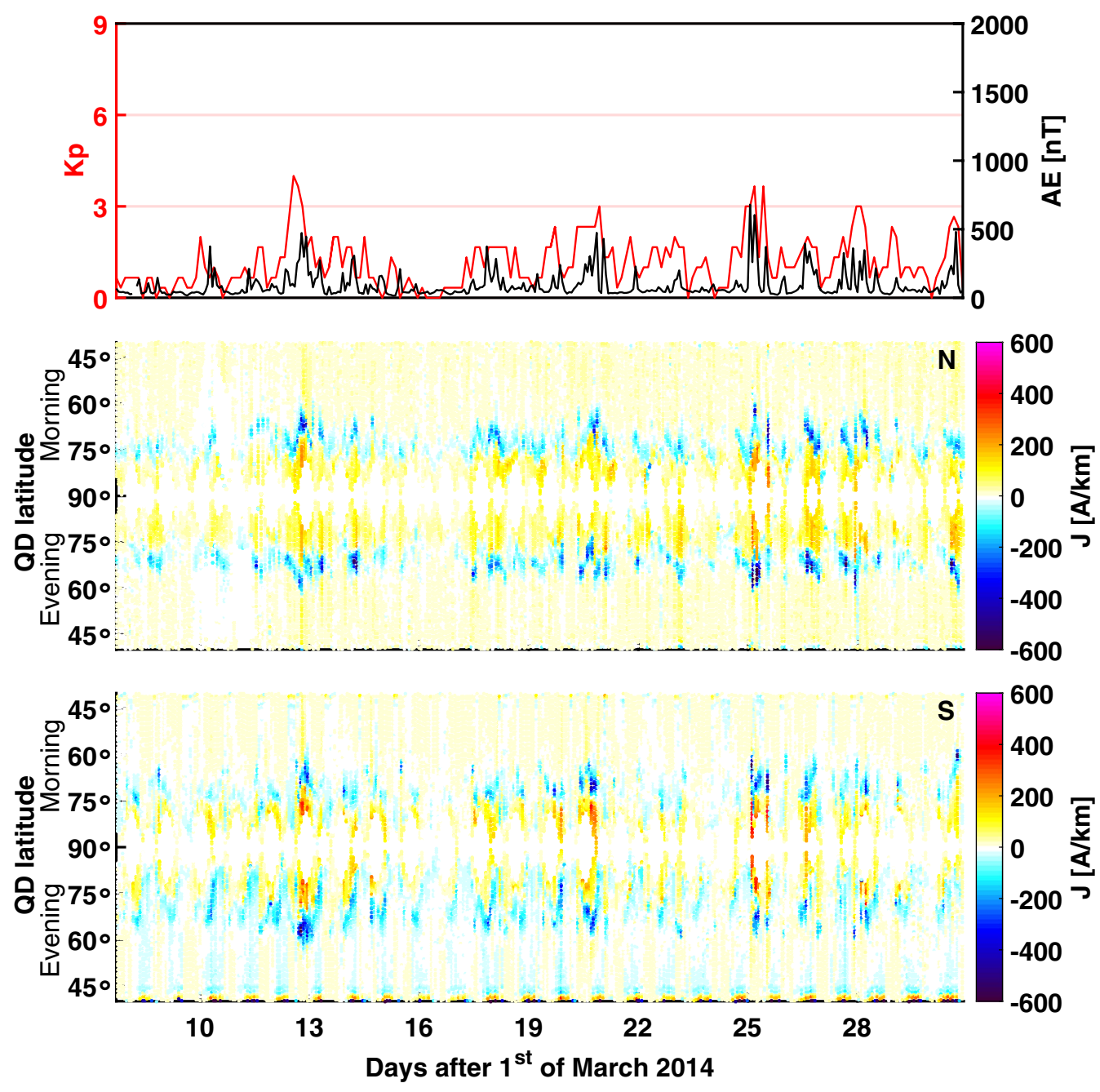

Fig. 4 Temporal development of the sheet current densities measured from satellite Alpha as a function of QD latitude and time (bottom two panels) along with the corresponding Kp and AE index values (top panel). The middle panel shows the temporal development for the Northern Hemisphere, while the bottom panel shows the temporal development for the Southern Hemisphere. The results are given for the period 8 to 31 March 2015 for satellite Alpha. A positive current is defined in the sunward direction

The intensities of the ionospheric currents estimated from this type of plot are a good way to show the development of a substorm event. We see, for example, how quiet periods are followed by a sudden intensification of the currents, and expansion of the auroral oval. An event like this marks the onset of a substorm (Akasofu 1964; McPherron 1991), here showing how plots like these can be used identify substorm events. Despite the rapid changes, especially during disturbed times, and the time of about 20 min that it takes for the satellite to cross one polar hemisphere, we still get a clear picture of the state of the ionospheric current system.

Figure 5 shows an example of a much more disturbed period, namely the period around the St. Patrick's day storm on 17 March 2015. This was the most severe geomagnetic storm of solar cycle 24 , with $\mathrm{Kp}$ indices as large as $8^{-}$, lasting for approximately $18 \mathrm{~h}$. Figure 5 clearly shows how the ionosphere is not only affected during the storm, but also for a long period thereafter. The onset of the storm is difficult to identify due to the effects of a smaller CME ejected from the sun just three days prior to the very large eruption that caused the storm on 17. We see, however, how the oval expands very far south during the storm, consistent with observations of aurora as far south as France.

The interhemispheric differences are larger for the period shown in Fig. 5, compared to that of Fig. 4. This might be due to the more rapid changes during disturbed times but could also indicate interhemispheric 

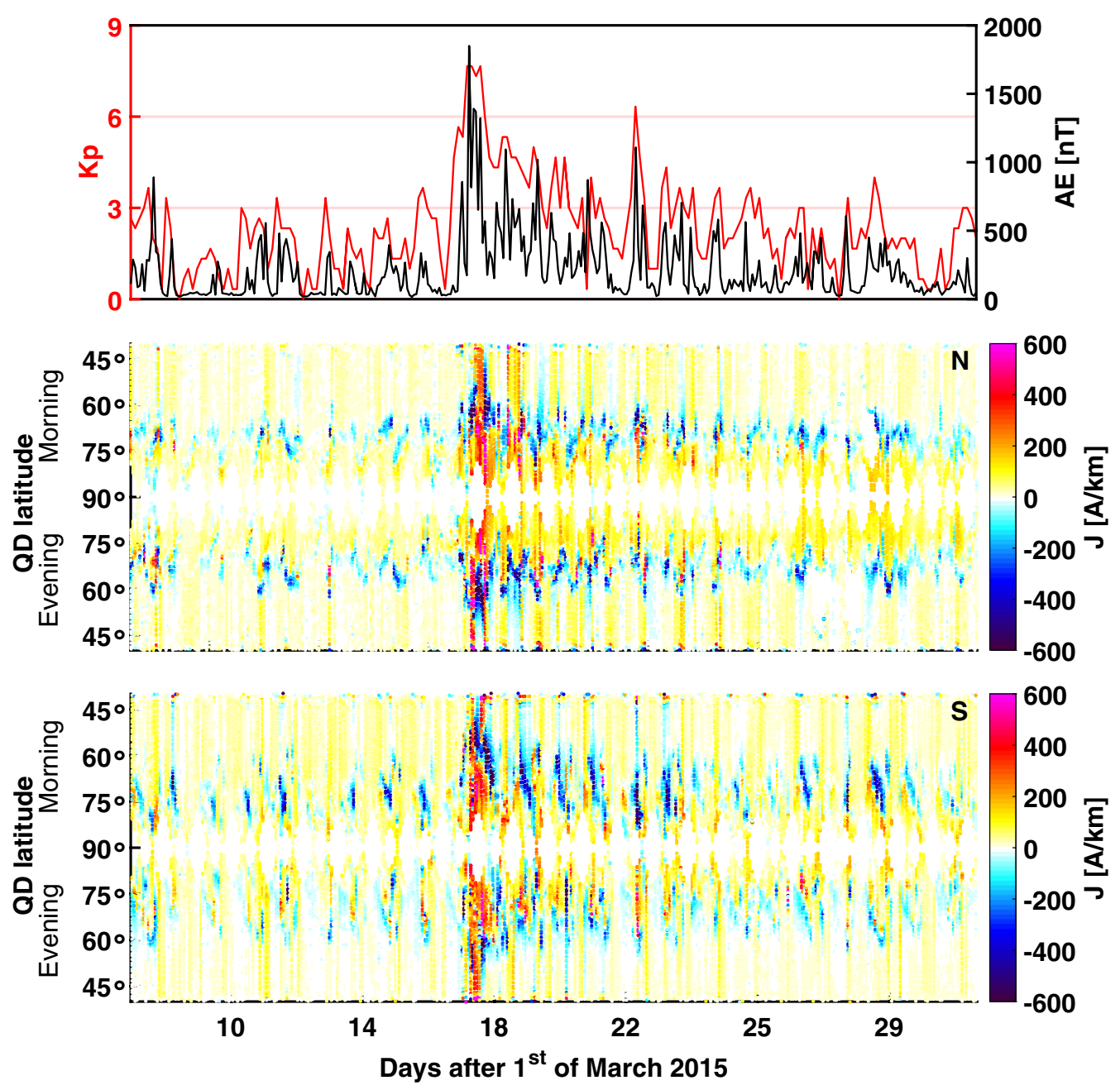

Fig. 5 Temporal development of the sheet current densities measured from satellite Alpha as a function of QD latitude and time (bottom two panels) along with the corresponding Kp and AE index values (top panel). The middle panel shows the results for the Northern Hemisphere, and the bottom panel shows the results for the Southern Hemisphere. The results are given for the period around the St. Patrick's day storm on 17 March 2015 (8 to 31 March 2015). A positive current corresponds to a sunward current

asymmetries. Nevertheless, in both cases we find very similar developments of the sheet current densities.

\section{Auroral Electrojet index}

We now compare our estimated sheet current densities with the AE index (Sugiura and Davis 1966). It is derived from geomagnetic variations in the horizontal component at 12 observatories along the northern auroral zone and aims at monitoring auroral electrojet activity (Baker 1985). AE provides an estimate of the combined strengths of Eastward and Westward electrojets. Data are found at the World Data Center for Geomagnetism (WDCG 2015).

Here, we compare AE with the total polar current, I,

$$
\boldsymbol{I}=\Delta \beta \int_{-50^{\circ}}^{50^{\circ}}|\boldsymbol{J}| \boldsymbol{d} \beta
$$

To enable comparison to the sheet current densities, we find the corresponding mean $\mathrm{AE}$ index for the period of the selected orbit. An example of this comparison is given in Fig. 6 for the Northern (top) and Southern (bottom) Hemisphere. The AE index is given in red (left $y$-axis), and the sheet current densities in black (right $y$-axis). An overall good correlation is found by visual inspection. The total polar current in black follows the $\mathrm{AE}$ index very nicely for both the Southern and Northern Hemisphere, despite the fact that they were derived using different methods and data sets (ground-based and spaceborn)

To give an idea of how well the two data series $\boldsymbol{A E}(t)$ and $\boldsymbol{I}(t)$ are correlated, we estimated the squared coherence of the two data series in dependence on frequency, $f$, and 

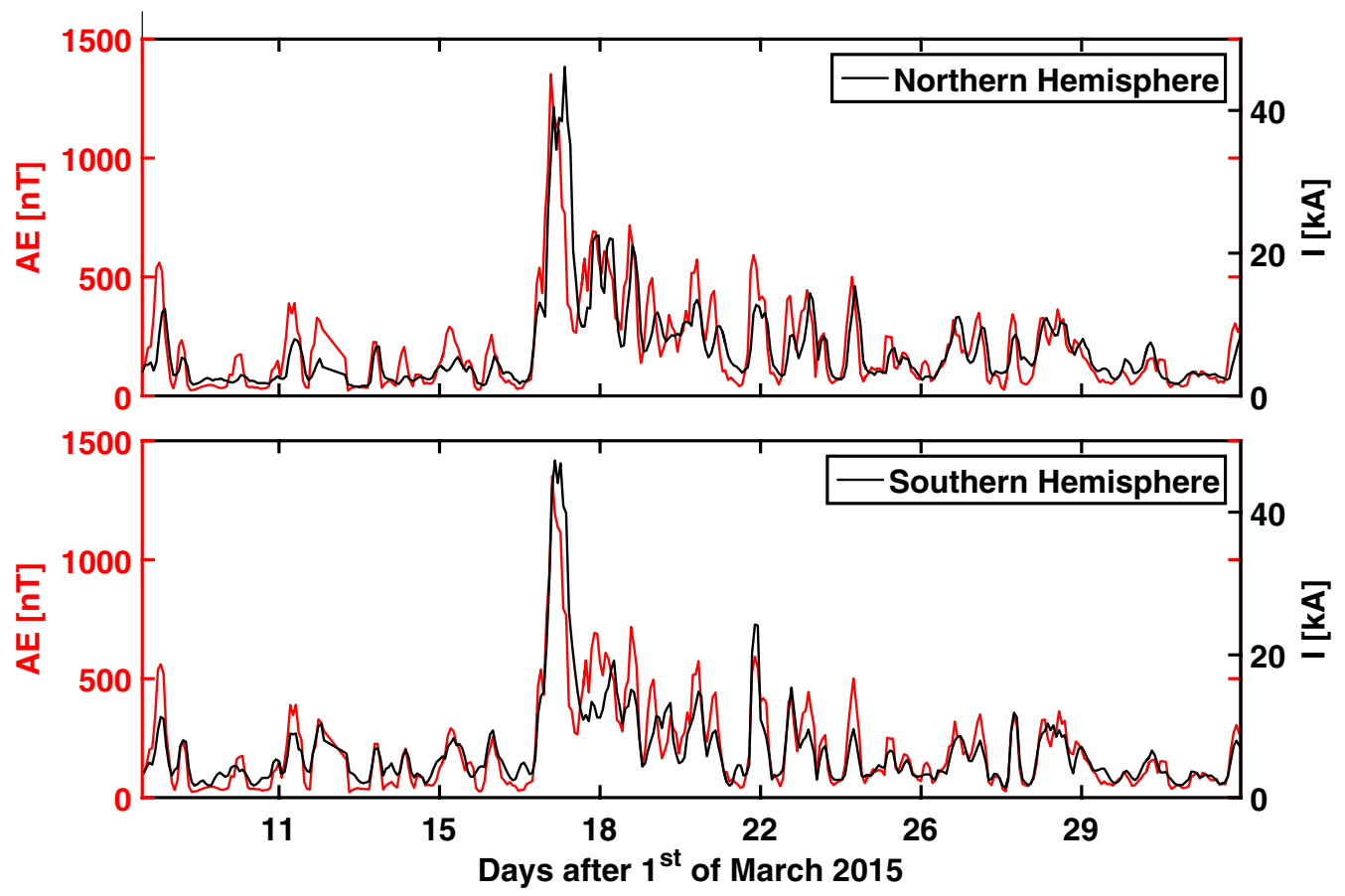

Fig. 6 Time series of AE index and the total polar current, I, for a period of 400 orbits (orbit number 7200 to 7799 ) from 7 March to 1 April 2015. The time series are smoothed with a 3-point running mean boxcar window for better comparison. The AE index is compared to the integrated time series of the sheet current densities for the Northern (top) and Southern (bottom) Hemisphere, respectively

corresponding period, $T=1 / f$, considering the 400 orbits (orbit number 7200 to 7799 ) from 7 March to 1 April 2015 given in Fig. 7. The black curve shows the coherence between the Northern Hemisphere sheet current densities and the AE index. The red curve shows the coherence between the Southern Hemisphere sheet current densities and the $\mathrm{AE}$ index, and the blue curve shows the coherence between the sheet current densities for the Northern and Southern Hemisphere. The interval studied here corresponds to a small selection of that presented in Fig. 5.

A Fourier transformation is used to determine $\boldsymbol{A E}(f)$ and the total polar currents $(I(f))$ in the frequency domain. Squared coherence between the two data series are found as a function of $T$. For all three cases, we find

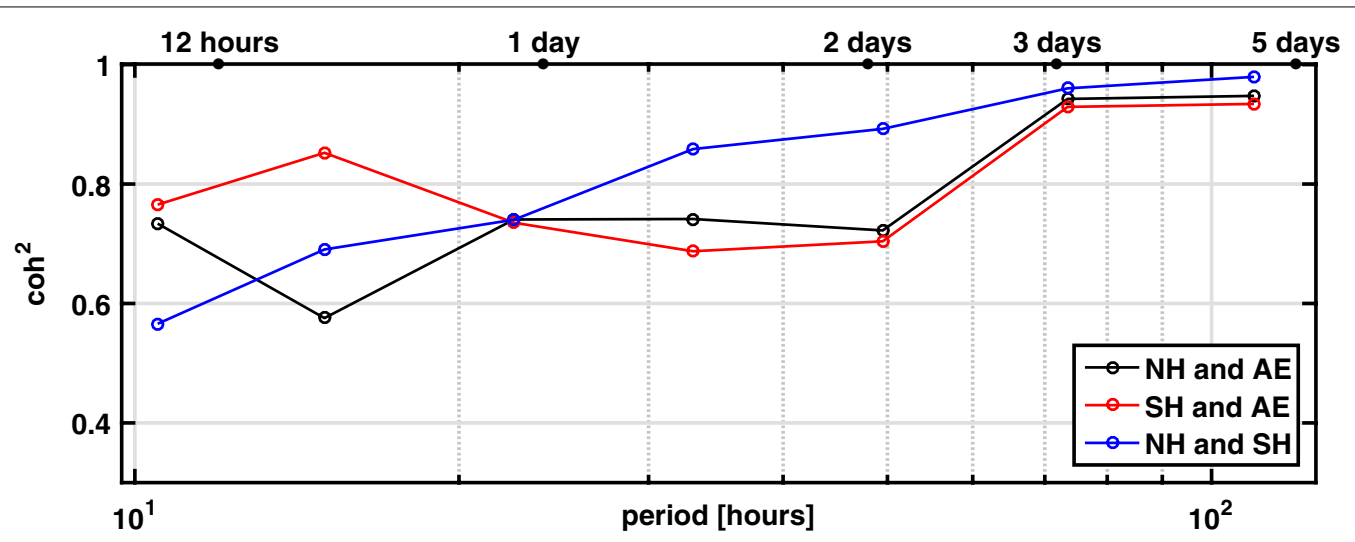

Fig. 7 Correlation between AE index and the total polar current, I, as a function of period. The correlation is found for the period of 400 orbits from 7 March to 1 April 2015. The black curve shows the coherence of the AE index with the Northern Hemisphere, the red shows the coherence of the AE index with the Southern Hemisphere, and the blue curve shows the coherence of the Northern and Southern Hemisphere time series as a function of the period of the signal 
that the squared coherency for daily variations, and all periods smaller than this, is relatively low, while the squared coherency for periods longer than two days is above 0.9. The interhemispheric coherency is in general a little higher than the coherency with the $\mathrm{AE}$ index. The high interhemispheric coherency shows a clear connection between the sheet current densities in the Northern and Southern Hemisphere confining the findings in Fig. 5.

The large coherence values between the total current and the AE index (black and red curve) supports the conclusion from the visual inspection of Figs. 4 and 5 of high correlation between currents (Northern and Southern Hemisphere) and the AE index.

\section{Intersatellites comparison}

The AE index provides a good measure of the auroral activity despite some limitations. Summing over longitudes removes any longitudinal dependence of the electrojet activity (see, e.g., Sugiura and Davis 1966). We show here how the integrated absolute values of the sheet current densities measured by satellite give comparable results to the AE index. With the Swarm satellite constellation it is not only possible to obtain a result comparable to the $\mathrm{AE}$ index, but also to estimate the longitudinal dependence of the electrojets by comparing the satellites Alpha/Charlie and Bravo. An example of the difference between satellite Alpha and Bravo is given in Fig. 8.

Visual inspection of the individual integrated time series of $J(\beta)$ for satellite Alpha and Bravo (top panel) shows high correlation between the two satellites. There are, however, also larger differences mainly during disturbed times. The longitudinal separation between satellites Alpha and Bravo was $24.5^{\circ}$ on 17 March 2015 at the equator. The differences are shown in the bottom panel as a function of both magnetic (QD) latitude and time. A positive difference indicates a stronger current for satellite Alpha. Comparing with the temporal development of Swarm Alpha (middle plot), we see that the largest differences are found in the auroral oval and during disturbed times (e.g. around $17 \mathrm{March}$ ). During disturbed periods, the differences (middle plot) are of comparable size to the sheet current densities found by Swarm Alpha (bottom plot). This, together with the longitudinal separation, shows that even smaller longitudinal length scales might be important in auroral electrojet currents during disturbed times.

Sheet current densities from 1000 orbits (orbit number 3000-4000) from Charlie are found to describe $97 \%$ of the variance from Alpha. This indicates that our technique is robust and insensitive to small changes in the data set.

\section{Induced currents}

Secondary, induced, currents in the electrically conducting Earth's upper mantle and lithosphere have until now been ignored. They can, however, easily be considered in our current model, by assuming a superconductor at depth, $d$, acting as a mirror for the primary, ionospheric, currents, placing the induced currents at radius, $r_{k}=a-2 d$. Induced currents are especially important for observations at ground level, such as directional drilling. The induced currents are implemented in the model by including an additional term (with opposite sign and a different radius of the currents) in the design matrix elements, $g_{n, k}$, presented in Eq. $3 . d$ is set to $125 \mathrm{~km}$, corresponding to a depth of the induced currents of $250 \mathrm{~km}$. The revised design matrix, including the effects of induction, has elements:

$$
\begin{gathered}
g_{n, k}=\frac{\mu_{0}}{2 \pi}\left[\frac{\xi_{n, k} \cos I_{n}+\eta_{n, k} \sin I_{n}}{\xi_{n, k}^{2}+\eta_{n, k}^{2}}-\frac{\left(\xi_{n, k}\right)_{j} \cos I_{n}+\left(\eta_{n, k}\right)_{j} \sin I_{n}}{\left(\xi_{n, k}\right)_{j}^{2}+\left(\eta_{n, k}\right)_{j}^{2}}\right] . \\
\left(\eta_{n, k}\right)_{j}=(a-2 d) \sin \left(\beta_{n}-\beta_{k}\right) \\
\left(\xi_{n, k}\right)_{j}=r_{n}-(a-2 d) \cos \left(\beta_{n}-\beta_{k}\right) .
\end{gathered}
$$

Figure 9 shows the magnetic field estimation (top), the residual magnetic field (middle), and the sheet current density (bottom) for orbit 6493 on 20 January 12:37 to 13:02 UT if induced currents are considered. Comparisons with Fig. 2 (without induced currents) reveal no significant changes in position or in the strength of the sheet current densities.

\section{Conclusions and outlook}

The magnetic perturbations due to ionospheric auroral electrojet current system were estimated by applying the line current model of Olsen (1996) to magnetic observations taken by the three satellites Alpha, Bravo, and Charlie of the Swarm satellite constellation. Sheet current densities were obtained using two different inversion methods: (1) minimization of an $L_{2}$ norm of the model parameters and a Huber-weighted misfit measure and (2) minimization of an $L_{1}$ norm of the second-order spatial differences of model parameters and a Huber-weighted misfit measure. The method is illustrated with examples from single orbits with model predictions that match very well the observations for both $L_{1}$ and $L_{2}$ norm models.

The $L_{1}$ norm solution is preferred since it leads to current profiles with weaker currents in non-polar regions. In addition, the distribution of model parameters possessed long tails, favouring the $L_{1}$ norm approach.

A study of the regularization parameter, $\alpha^{2}$, revealed that a common choice was possible for all tested orbits. This indicates a robust model, not sensitive to disturbance level or other orbit sensitive factors. The tests also indicate that the results are fairly insensitive to the exact 

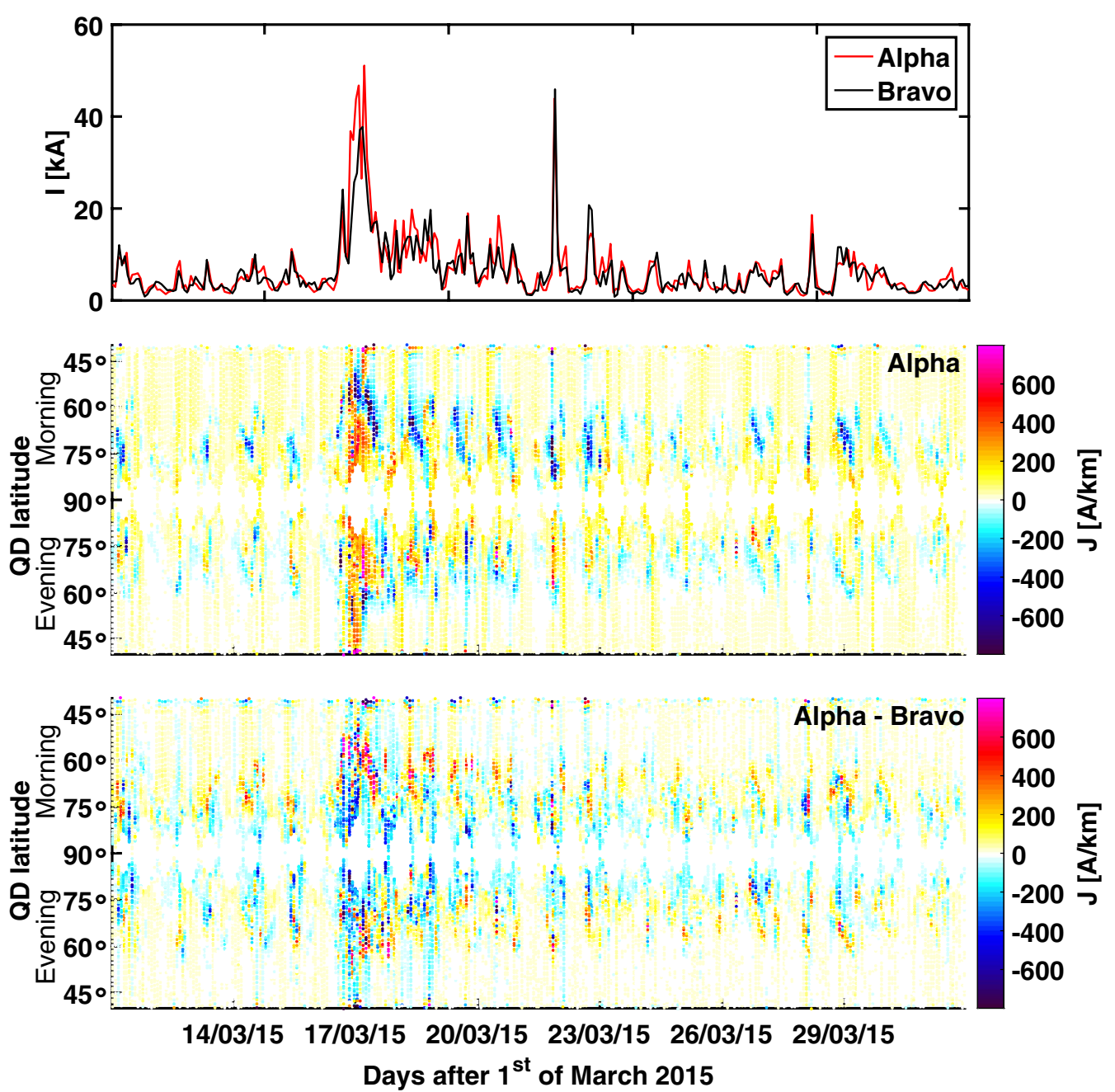

Fig. 8 Temporal development of the sheet current densities and their differences for satellite Alpha and Bravo for the Northern Hemisphere in the period 11 to 31 March 2015. The top panel shows the integrated time series of the sheet current densities for satellite Alpha and Bravo for 300 orbits. The middle panel shows the sheet current densities of Swarm Alpha as a function of magnetic latitude and time (corresponding to middle plot of Fig. 5), and the bottom panel, the temporal development of their differences. Positive difference follows times with largest sheet current densities found by Swarm Alpha

choice of $\alpha^{2}$. Model robustness was tested by comparing results obtained with data from the side-by-side flying satellites Alpha and Charlie: currents derived with Charlie describe more than $97 \%$ of those derived from Alpha.

Investigations of the time dependence of the sheet current densities for the two hemispheres showed a clear difference between disturbed and quiet times. They also revealed a very high consistency between the two hemispheres, with largest differences occurring during disturbed periods which are attributed to the fast changes in the ionospheric current system during these times. The visual consistency is backed up by a squared coherence analysis that revealed a value of more than 0.9 for periods larger than two days.
Comparison to the $\mathrm{AE}$ index also showed a very high squared coherence value $(\approx 0.9)$ for periods longer than two days for the Northern and Southern Hemisphere.

The line currents in this study are placed perpendicular to the satellite track. This can lead to a systematic underestimation of the sheet current densities, dependent on the angle with the actual current. The effect will therefore be largest in the region around the poles, though it will differ from orbit to orbit, dependent on the satellite track. A more accurate approach would be to assume the currents to be parallel to magnetic latitude.

The results pave the way for further research possibilities, such as combining the height-integrated current density estimates with measurements from the Swarm 

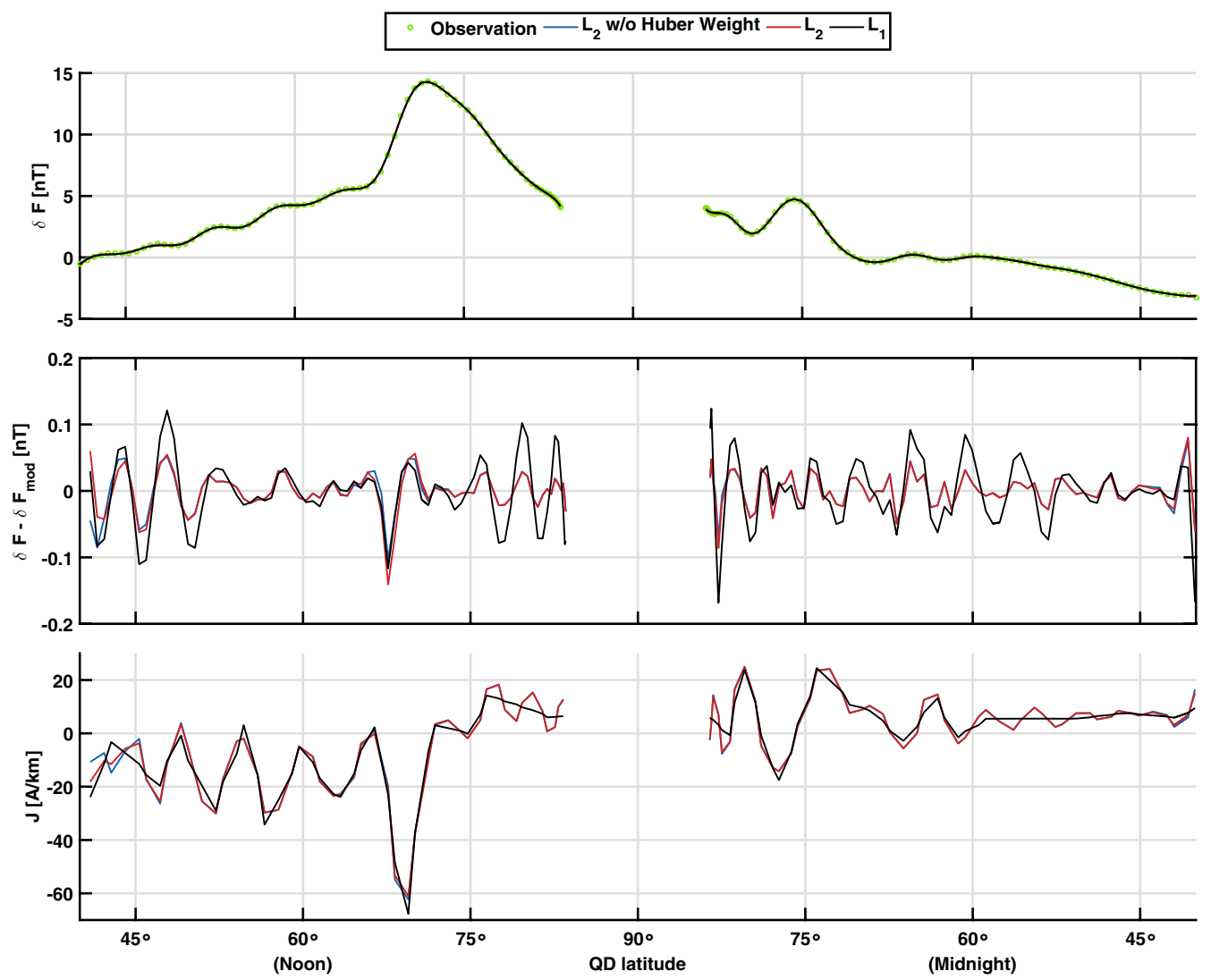

Fig. 9 Corresponding to Fig. 2 for Swarm Alpha, orbit 6493 on 20.01.2015 12:37 to 13:02 UT, including induced currents. The top panel shows observations, $\delta F$, in green along with the model predictions, $\delta F^{\text {mod }}$, for three solution methods, $L_{1}$ norm (black), $L_{2}$ norm (red), and $L_{2}$ norm without Huber-weighted misfit (blue). The middle panel shows the model residuals, $\Delta \delta F$, for the same inversion methods, and the bottom panel shows the corresponding sheet current densities, J, again for all three methods. The sheet current densities are found using $\alpha^{2}=6.5 \cdot 10^{-15} \frac{\mathrm{nT}^{2}}{\mathrm{~A}}$ for the $L_{1}$ norm solution and $\alpha^{2}=1 \cdot 10^{-26} \frac{\mathrm{nT}^{2}}{\mathrm{~A}^{2}}$ for the $L_{2}$ norm solutions. A positive current flows from midnight to noon (sunward)

Electric Field Instrument to derive height-integrated Hall conductivities.

Overall, we found that the line current model provides useful estimates of the polar ionospheric sheet current densities. The robustness of the method and the fact that the method worked for all tested orbits reveal a clear opportunity for automatic application and near-real-time estimations of the ionospheric sheet current densities.

\section{Authors' contributions}

CDAa coded the model, carried out the data analysis, and drafted the manuscript. NO and CCF participated in key discussions of the model and data analysis. All authors read and approved the final manuscript.

\section{Acknowledgements}

We would like to thank ESA for providing prompt access to the Swarm L1b data. This project was supported by The Research Council of Norway through the PETROMAKS research programme. The financial support is gratefully acknowledged.

\section{Competing interests}

The authors declare that they have no competing interests.
Received: 8 February 2016 Accepted: 6 July 2016

Published online: 05 August 2016

\section{References}

Akasofu S-I (1964) The development of the auroral substorm. Planet Space Sci 12(4):273-282. doi:10.1016/0032-0633(64)90151-5

Amm O (1997) lonospheric elementary current systems in spherical coordinates and their application. J Geomagn Geoelectr 49(7):947-955

Aster RC, Borchers B, Thurber CH (2005) Parameter estimation and inverse problems, 1st edn. Elsevier, Amsterdam

Baker D (1985) Statistical analyses in the study of solar wind-magnetosphere coupling. In: Technical report, Los Alamos National Laboratory, NM (USA)

Constable CG (1988) Parameter estimation in non-Gaussian noise. Geophys J 94:131-142

Ekblom $H$ (1987) The $L_{1}$-estimate as limiting case of an $L_{p}$-or Huber-estimate. In: Dodge $Y\left(\right.$ ed) Statistical data analysis based on the $\mathrm{L}_{1}$-norm and related methods. Elsevier, Amsterdam, pp 109-116

Farquharson CG, Oldenburg DW (1998) Non-linear inversion using general measures of data misfit and model structure. Geophys J Int 134(1):213227. doi:10.1046/.1365-246x.1998.00555.x

Finlay CC, Olsen N, Tøffner-Clausen L (2015) DTU candidate field models for IGRF-12 and the CHAOS-5 geomagnetic field model. Earth Planets Space 67(1):1-17 
Finlay CC, Olsen N, Kotsiaros S, Gillet N, Tøffner-Clausen L (2016) Recent geomagnetic secular variation from Swarm and ground observatories in the CHAOS-6 geomagnetic field model. Earth Planets Space. doi:10.1186/ s40623-016-0486-1

Friis-Christensen E, Kamide Y, Richmond AD, Matsushita S (1985) Interplanetary magnetic field control of high-latitude electric fields and currents determined from greenland magnetometer data. J Geophys Res Space Phys 90(A2):1325-1338. doi:10.1029/JA090iA02p01325

Friis-Christensen E, Lühr H, Knudsen D, Haagmans R (2008) Swarm-an earth observation mission investigating geospace. Adv Space Res 41(1):210216. doi:10.1016/j.asr.2006.10.008

Hansen PC (1992) Analysis of discrete ill-posed problems by means of the L-curve. SIAM Rev 34(4):561-580

Huber PJ (1964) Robust estimation of a location parameter. Ann Math Stat 35(1):73-101. doi:10.1214/aoms/1177703732

Jackson A, Constable C, Gillet N (2007) Maximum entropy regularization of the geomagnetic core field inverse problem. Geophys J Int 171(3):995-1004. doi:10.1111/j.1365-246X.2007.03530.x

Juusola L, Amm O, Viljanen A (2006) One-dimensional spherical elementary current systems and their use for determining ionospheric currents from satellite measurements. Earth Planet Space 58:667-678

Kamide Y, Richmond AD, Matsushita S (1981) Estimation of ionospheric electric fields, ionospheric currents, and field-aligned currents from ground magnetic records. J Geophys Res 86(A2):801-813. doi:10.1029/ JA086iA02p00801

Lesur V, Wardinski I, Hamoudi M, Rother M (2010) The second generation of the GFZ reference internal magnetic model: GRIMM-2. Earth Planets Space 62:765-773. doi:10.5047/eps.2010.07.007

Liu H, Lühr H (2005) Strong disturbance of the upper thermospheric density due to magnetic storms: CHAMP observations. J Geophys Res Space Phys 110(A9). doi:10.1029/2004JA010908

McPherron RL (1991) Physical processes producing magnetospheric substorms and magnetic storms. In: Jacobs JA (ed) Geomagnetism. Academic Press Ltd, London, pp 593-739

Menke W (2012) Geophysical data analysis: discrete inverse theory, MATLAB edition, 3rd edn. Elsevier, Amsterdam

Moretto T, Olsen N, Ritter P, Lu G (2002) Investigating the auroral electrojets with low altitude polar orbiting satellites. Ann Geophys 20(7):1049-1061

Olsen N (1996) A new tool for determining ionospheric currents from magnetic satellite data. Geophys Res Lett 23(24):3635-3638. doi:10.1029/96GL02896

Olsen N, Moretto T, Friis-Christensen E (2002) New approaches to explore the earth's magnetic field. J Geodyn 33(1-2):29-41 (earth's Gravity and Magnetic Fields from Space)
Olsen N, Lühr H, Finlay CC, Sabaka TJ, Michaelis I, Rauberg J, Tøffner-Clausen L (2014) The CHAOS-4 geomagnetic field model. Geophys J Int 197(2):815-827

Pirjola R, Kauristie K, Lappalainen H, Viljanen A, Pulkkinen A (2005) Space weather risk. Space Weather 3(2). doi:10.1029/2004SW000112

Poedjono B, Beck N, Buchanan A, Borri L, Maus S, Finn CA, Worthington EW, White T et al (2013) Improved geomagnetic referencing in the arctic environment (russian). In: SPE arctic and extreme environments technical conference and exhibition, society of petroleum engineers

Richmond A, Lu G, Emery B, Knipp D (1998) The AMIE procedure: prospects for space weather specification and prediction. Adv Space Res 22(1):103112. doi:10.1016/S0273-1177(97)01108-3 (solar-Terrestrial Relations: Predicting the Effects on the Near-Earth Environment)

Richmond AD (1995) lonospheric electrodynamics using magnetic apex coordinates. J Geomagn Geoelectr 47(2):191-212. doi:10.5636/jgg.47.191

Ritter P, Viljanen A, Lühr H, Amm O, Olsen N (2003) lonospheric currents from champ magnetic field data comparison with ground based measurements. In: Reigber C, Lühr H, Schwintzer P (eds) First CHAMP mission results for gravity, magnetic and atmospheric studies. Springer, Berlin, pp 347-352. doi:10.1007/978-3-540-38366-6 50

Ritter P, Lühr H, Viljanen A, Amm O, Pulkkinen A, Sillanpää I (2004) Ionospheric currents estimated simultaneously from champ satelliteand image ground-based magnetic field measurements: a statistical study at auroral latitudes. Ann Geophys 22(2):417-430. doi:10.5194/angeo-22-417-2004

Sabaka TJ, Olsen N, Purucker ME (2004) Extending comprehensive models of the Earth's magnetic field with Ørsted and CHAMP data. Geophys J Int 159:521-547. doi:10.1111/j.1365-246X.2004.02421.x

Sabaka TJ, Olsen N, Tyler RH, Kuvshinov A (2015) CM5, a pre-Swarm comprehensive magnetic field model derived from over 12 years of CHAMP, Ørsted, SAC-C and observatory data. Geophys J Int 200:1596-1626. doi:10.1093/gji/ggu493

Sugiura M, Davis T (1966) Auroral electrojet activity index AE and its universal time variations. J Geophys Res 71:785-801

Tikhonov AN (1963) Solution of incorrectly formulated problems and the regularization method. In: Soviet Math. Dokl., vol 4, pp 1035-1038, english translation of Dokl. Akad. Nauk. SSSR, 151 (1963), pp 501-504

Vennerstrom S, Moretto T (2013) Monitoring auroral electrojets with satellite data. Space Weather 11(9):509-519. doi:10.1002/swe.20090

WDCG (2015) World Data Center for Geomagnetism (WDCG), Kyoto University. http://wdc.kugi.kyoto-u.ac.jp/aeasy/index.html

\section{Submit your manuscript to a SpringerOpen ${ }^{\odot}$ journal and benefit from:}

- Convenient online submission

- Rigorous peer review

Immediate publication on acceptance

- Open access: articles freely available online

- High visibility within the field

- Retaining the copyright to your article

Submit your next manuscript at springeropen.com 